\title{
Shape and Level Bottles Detection Using Local Standard Deviation and Hough Transform
}

\author{
Nor Nabilah Syazana Abdul Rahman ${ }^{1}$, Norhashimah Mohd Saad ${ }^{2}$, Abdul Rahim Abdullah ${ }^{3}$ \\ ${ }^{1,2}$ Faculty of Electronic and Computer Engineering, Universiti Teknikal Malaysia Melaka, Malaysia \\ ${ }^{2,3}$ Center for Robotics and Industrial Automation, Universiti Teknikal Malaysia Melaka, Malaysia \\ ${ }^{3}$ Faculty of Electrical Engineering, Universiti Teknikal Malaysia Melaka, Malaysia
}

\begin{tabular}{l} 
Article Info \\
\hline Article history: \\
Received Feb 23, 2018 \\
Revised Jul 19, 2018 \\
Accepted Jul 22, 2018 \\
\hline Keyword: \\
Automated inspection \\
Hough transform \\
Level detection \\
Local standard deviation \\
Shape detection
\end{tabular}

\begin{abstract}
This paper presents shape and level analysis using local standard deviation and Hough transform technique to detect the shape and level of the bottle. A 155 sample images are used as a test product to detect shape and level. Local standard deviation is used contrast gain technique to segment the shape of the bottle by enhancing the contrast of the image. The ratio of the area is calculated from the extent parameter. The maximum and minimum water level is created by using Hough transform technique to identify the level of the water. Decision tree is applied to classify the shape and level of the bottle either good or defect condition. From experimental result, 97\% and 93\% accuracy of shape and level is achieved which shows that the proposed analysis technique is potential to be applied for beverages product inspection system.
\end{abstract}

Copyright $\odot$ 2018Institute of Advanced Engineering and Science. All rights reserved.

\section{Corresponding Author:}

Norhashimah Mohd Saad,

Faculty of Electronic and Computer Engineering,

Universiti Teknikal Malaysia Melaka,

Hang Tuah Jaya, 76100 Durian Tunggal, Melaka, Malaysia.

Email: norhashimah@utem.edu.my

\section{INTRODUCTION}

Industries such as textile, semiconductor, food and beverages have agreed that a system based approach is more efficient and sustainable to be applied in manufacturing process [1]. Visual inspection is categorized into two, which are manual inspection and automatic inspection. Manual inspection is commonly used human as inspector for product quality [2]. The study made by [3] have shown the manual inspection performed by human operator is inefficient and consume more time due to working condition. The task given by industry is repetitive and difficult to do if no proper training is conducted. An automatic inspection seems to be better compared to manual inspection. For these reason, the application of an automated inspection system for detection and classification of defect is desirable [4]. The application of an automated inspection has reduced execution time, computational cost and high percentage error free [5].

Some reviews are made from previous research to compare the inspection system technique. Researcher in [6] proposed shape detection using a partial erosion-based technique to automate the shape segmentation process of plastic bottle. The proposed technique involved morphological and erosion process to segment the bottle shape and extract the features from the segmented image. The accuracy of the proposed technique is more than $80 \%$. However, the partial erosion-based technique is inefficient and give bad effect to the image when the partial erosion is set less than $50 \%$ or more than $100 \%$. The statistical histogram based Fuzzy C-means (SHFCM) is suggested by [3] to identify the apple defect for fruit quality inspection. SHFCM is used to classify the healthy and defect apple. The experimental result shows the accuracy achieved by healthy apple is $96 \%$ and defect apple is $91 \%$. Nevertheless, SHFCM is the combination of the 
statistical histogram and fuzzy c-means which makes the algorithm more complex and prone to misclassified healthy and defect apple.

For level detection, liquid level inspection system has been developed by [7] based on infinite symmetrical edge filter (ISEF) detection technique to replace traditional quality inspection perform by a human operator. The process includes image cropping and normalizing, filtering and detecting. The analysis results show the proposed technique is better detection of overfill and underfill for liquid level. The proposed technique is not suitable for the quality image because it will over segment the image. Furthermore, [8] proposed feature extraction and edge detection algorithm to inspect fill level and cap in bottling machine. Classification of liquid level and cap closure is done by using neural network $(\mathrm{NN})$ technique. The proposed technique proves that it can inspect liquid level and cap of bottle accurately. Nevertheless, lower or empty liquid level cannot be detected and too many pre-processing techniques will reduce the image quality.

Based on literature, two main problems that occur during beverage bottle quality inspection are highlighted. First, the used of manual inspection is highly prone to human error. Second isan inappropriate image processing technique chosen is highly prone to under or over segmentation, on the other hand lead to loss of precision. Hence, this paper proposed a new analysis technique of shape and level for beverages quality inspection system using local standard deviation and Hough transform. The contrast enhancement technique provide by LSD will give different contrast level in order to prevent from under and over segmentation of the image. Thus, the shape of the image can be segmented accurately compared to previous technique,andpractically appropriated for industrial real-time inspection system [9]. Meanwhile, Hough transform is proposed because of its robustness to noise and capability to detect line without enough information, in accordance achieved betteraccuracy in detecting multiple object compared to others previous technique [10].

\section{RESEARCH METHOD}

The analysis of shape and level defect detection is done using the MATLAB software. The framework analysis of shape and level detection is shown in Figure 1. A 100 sample images is used for shape defect detection and 55 sample images for level defect detection. The sample image is captured using Canon D3100 digital camera with 12 megapixels. Then, the captured image is pre-processed to eliminate the noise and enhance the brightness of the image for further analysis.

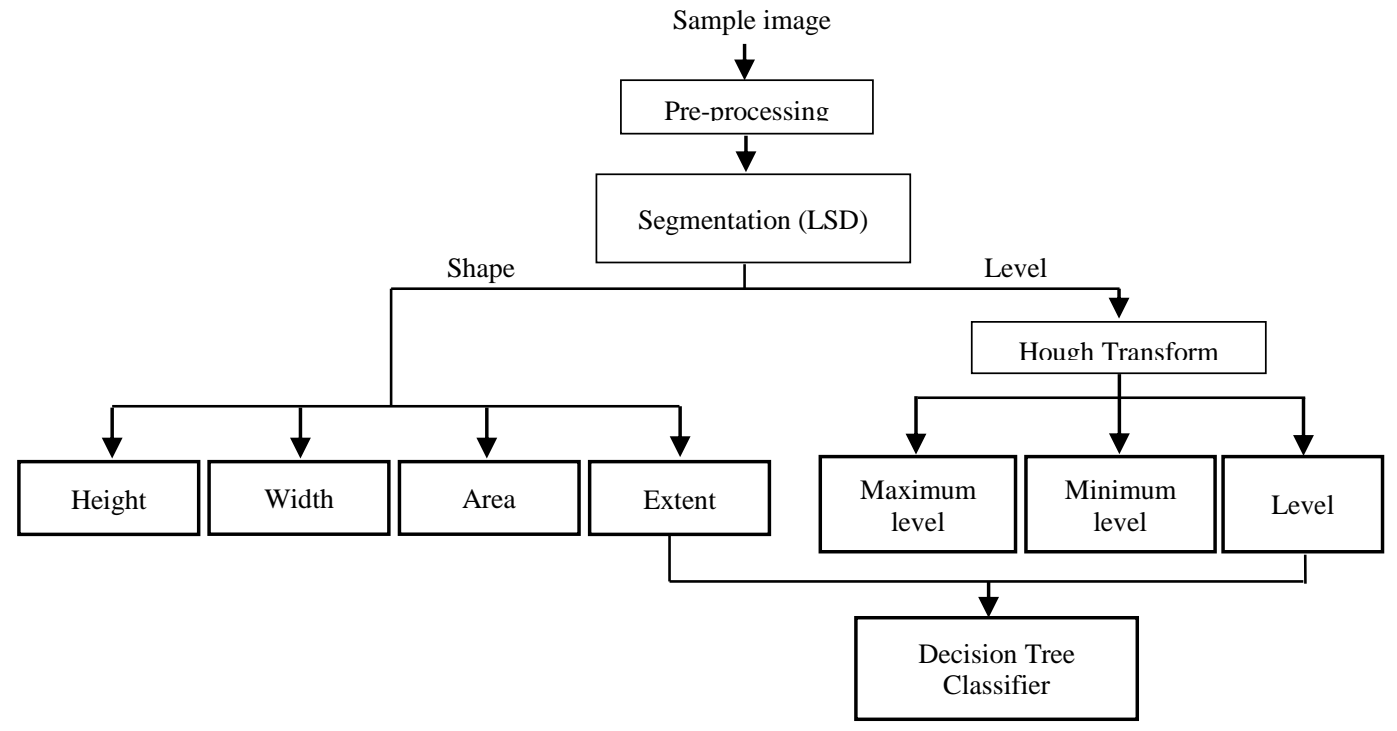

Figure 1. Framework analysis

\subsection{Pre-Processing}

Pre-processing is a common operation to standardized the image in order to minimize the complexity of the algorithm [11]. The noise occur on the image may give complexity during segmentation process. Besides, the background image is given similar gray level values with certain bottle structures. Therefore, pre-processing is carried out to correct the image for furtheranalysis. 


\subsection{Segmentation using Local Standard Deviation}

The Local Standard Deviation (LSD) is part of the Adaptive Contrast Enhancement (ACE) function to segment the shape of the image. ACE is known as an unsharp masking technique where the function is applied at the unsharp mask to amplify the image which haslow-frequency components. Besides, ACE will adjust the contrast gain of the image into suitable value. The general equation [12] is defined as

$$
f(i, j)=m_{x}(i, j)+G(i, j)\left[x(i, j)-m_{x}(i, j)\right]
$$

where $m_{x}=(i, j)$ is the local mean, $G=(i, j)$ is the contrast gain and $x=(i, j)$ is gray scale value at any point of the image pixel.

In the ACE, the function of LSD is to enhance the contrast gain of the image from low to high and high to low [13]. The ringing and noise result may occur if the contrast gain is set manually. ACE function is inversely proportional to LSD make the background image can be identified and eliminated at the edge of bottle. The unwanted contrast gain in the image is eliminated by enhancing the contrast gain from low to high using LSD function [14]. The function of LSD is expressed as

$$
f(i, j)=m_{x}(i, j)+\frac{D}{\sigma_{x}(i, j)}\left[x(i, j)-m_{x}(i, j)\right]
$$

where $D$ is a constant value for contrast and $\sigma_{X}(i, j)$ is a function of LSD. From equation (1), the contrast gain is automatically controlled by the $\sigma_{X}(i, j)$. Therefore, for the region that has small $m_{x}=(i, j)$ leads to an unexpectedly high value of LSD and vice versa.

\subsection{Hough Transform}

Hough transform is applied for the binary image to detect the lines of the water level in the bottle. The white pixels in the image has created a locus of reference points that accumulated in accumulator array of Hough space. The voting process is involved for line detection where every pixel in the image votes for all possible line corresponding to reference point. A line is passing through several feature points and maximum point which have indicated as reference points in the Hough space. Generally, $(x, y)$ space is converted into $(\rho, \theta)$ space which can be expressed as

$$
\rho=x \cos \theta+y \sin \theta
$$

where $\rho$ is the length of the line from the origin. $\theta$ is the angle of the $\rho$ with respect to the $\mathrm{x}$ direction. Figure 2 shows the conversion of $x y$ space into $(\rho, \theta)$ space where the lines are created based on several points in order to find the maximum and minimum value.

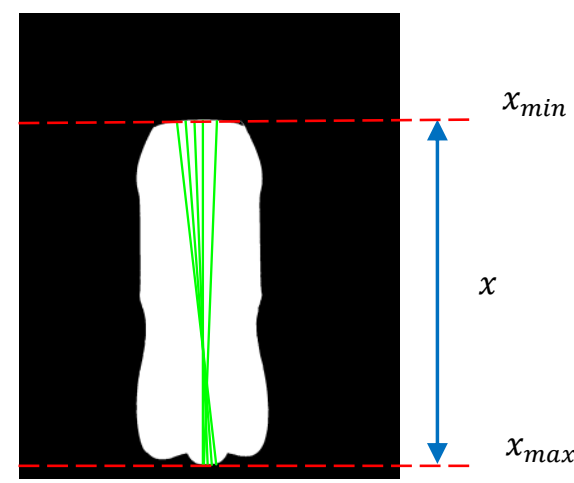

Figure 2. Created lines using Hough transform 


\subsection{Feature Extraction}

In image analysis, feature extraction is required to be input for classification process. A set of features are extracted from the segmented images based on the shape and level. For the shape features, parameters such as height, width, area and extent are extracted. Meanwhile, the water level feature is extracted by finding the maximum and minimum value of water level. These features are important to classify the defect of the image.

\subsubsection{Shape Detection}

Four parameters are extracted in shape features, which are height, width, area and extent. Figure 3 shows the dimension of the bounding box in the binary image that is used to obtain the area of the bottle. Based on Figure 3, the height of the image is determined as 4160 pixels while the width is obtained as 3120 pixels. The area of the bottle is calculated based on the white pixel. In determining the extent value, the area of the object is divided with the area of the bounding box in order to classify the shape defect of the bottle. The extent value is calculated as follows

$$
\text { Extent }=\frac{\text { Area of object }}{\text { Area of bounding box }}
$$

The white pixel has represented the shape of the bottle while the black pixel is the background image as illustrated in Figure 3. Hence, the area of the object is determined by the value of the white pixel. In addition, the area of the bounding box is obtained from multiplication value of height and width that can be expressed as

$$
\text { Area of bounding box }=\text { height } x \text { width }
$$

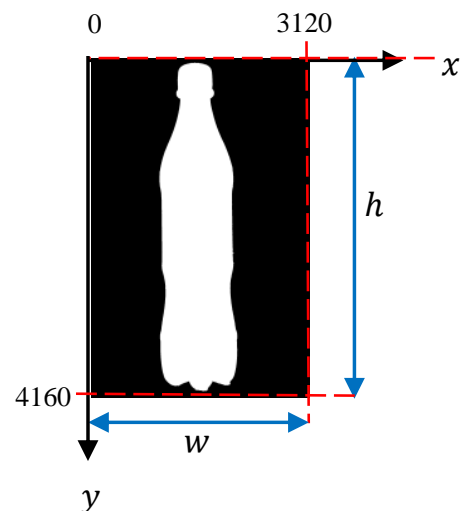

Figure 3. Bounding box dimension

\subsubsection{Level Detection}

The water level feature has been extracted using Hough transform that created the tangent line for the measurement distance between the maximum and minimum values. Figure 4 shows the maximum and minimum line of the water level. Each level of water has been labeled that is illustrated in Figure 4.

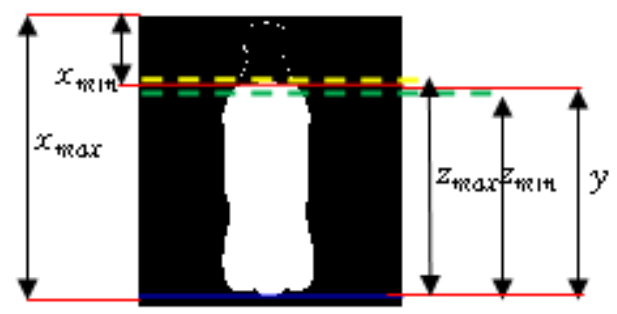

Figure 4. Label for level of water 
The level of water is classified in three conditions, whichare good, overfilled and underfilled. From Figure 4 , the level of water, $y$ is calculated from the distance between maximum $\left(x_{\max }\right)$ and minimum $\left(x_{\min }\right)$ as formulated in equation (6). The $z_{\max }$ and $z_{\min }$ value are set based on the good condition value of the bottle. The condition is good when the level is between the $z_{\max }$ and $z_{\min }$, If the level is above than $z_{\max }$, it will consider as overfilled. Otherwise, if the level is below than $z_{\min }$, the condition is underfilled.

$$
y=x_{\max }-x_{\min }
$$

\subsection{Decision Tree Classifier}

In order to classify the defect of shape and level, decision tree is implemented in this study. The tree consists of branches, nodes and leaves that are used for the classification process. Branches of the tree are indicated as values while nodes are represented as the name of data. The labels of the classare shown by the leaf [15]. Figure 5illustrates the training set of decision tree process that includes shape and level classification. Based on Figure 5, the decision tree model learns the extracted features of the bottle, which are extent valueand level. If the feature is the extent value, the shape of the bottle will be classified either good or defect condition. When the feature is the level value, the water level is classified into three conditions such as good, overfilled and underfilled.

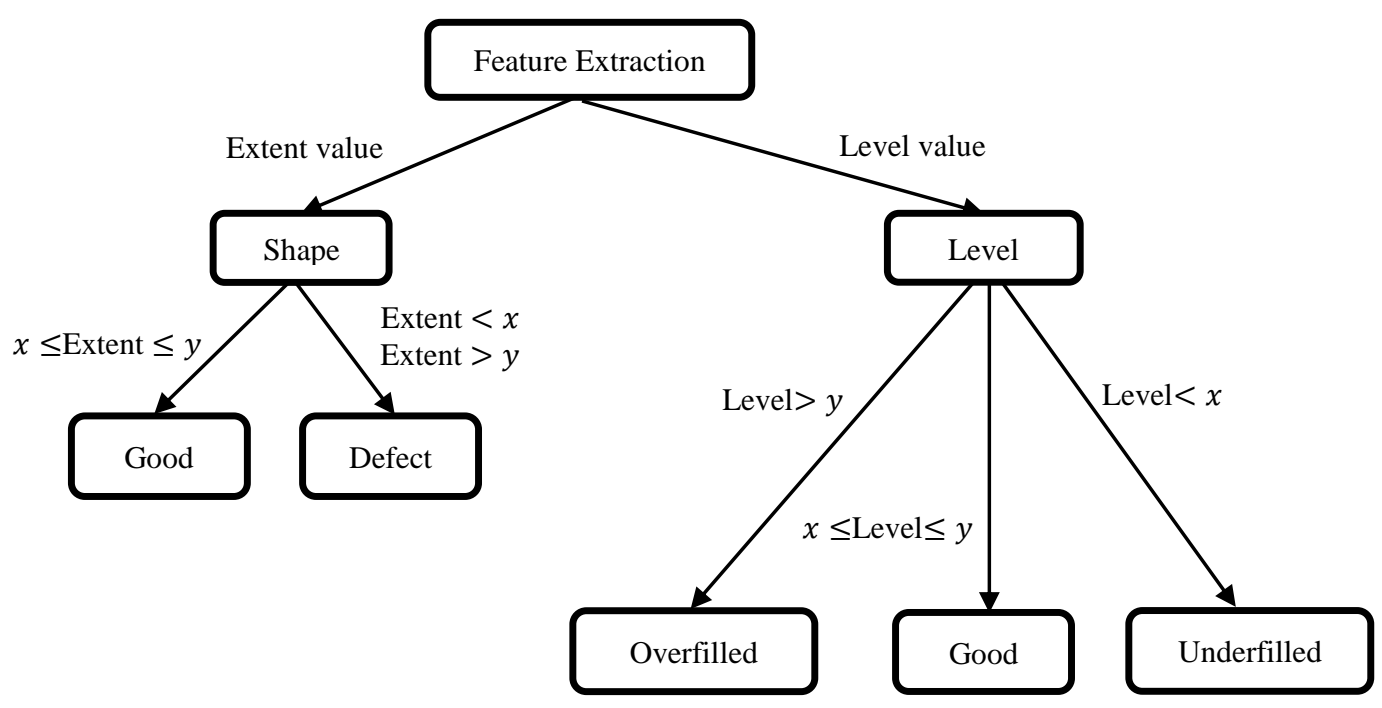

Figure 5. Decision tree process

\section{RESULTS AND ANALYSIS}

Figure 6 shows four different colors of bottles with the same size and level are used in this analysis as reference image.

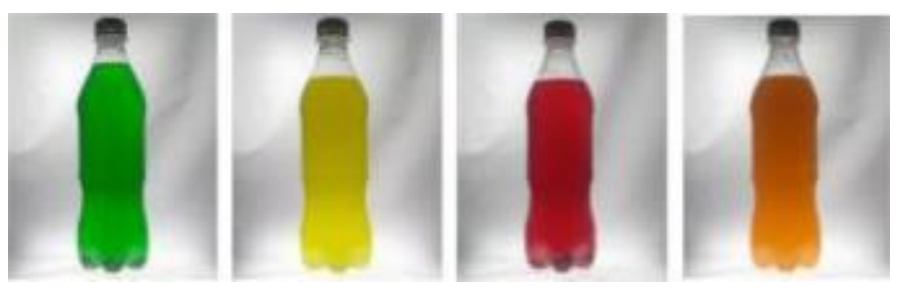

Figure 6. Reference image

Local standard deviation (LSD) uses contrast enhancement technique to segment the shape of the bottle. The segmentation of the bottle is applied to classify the shape condition either good or defect. Figure 
7(a) to Figure 7(d) shows the shape analysis process for the defect bottle. The process is started with the conversion of red, green and blue (RGB) color image in Figure 7(a) into a grayscale image in Figure 7(b). The conversion process is applied to reduce the complexity from 3-dimensional pixel into 2-dimensional pixel. The grayscale image is then transformed into standard deviation image as shown in Figure 7(c). In this process, standard deviation algorithm is applied to segment the shape of the bottle by separating the bottle and the background image.

The background noise at the edge of the bottle can be removed by increasing the value of LSD resulting the reduction of contrast gain of the image. Therefore, the shape of the bottle is outlined using the white line to separate between the shape of the bottle and the background image. The standard deviation image is converted into a binary image as represented in Figure 7(d), in which the shape of the bottle is presented in white pixel (1) while the background image in the black pixel (0).

Finally, the parameters extracted from Figure 7(d) are used as an input for the classification process. Four parameters namely height, width, area, and extent are extracted from the good and defect shape images. The extent parameter is calculated based on the ratio of the bottle area and the bounding box area that stated in the equation (4). For the area of bounding box, equation (5) is used by multiplying the height and width value. As expected, both sample images have similar area of bounding box but different value of the bottle area. The area value for good bottle was higher compared to defect bottle which then influenced the extent value.

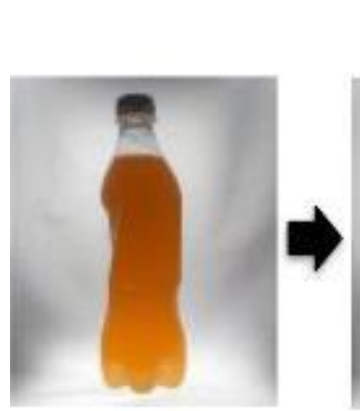

(a)

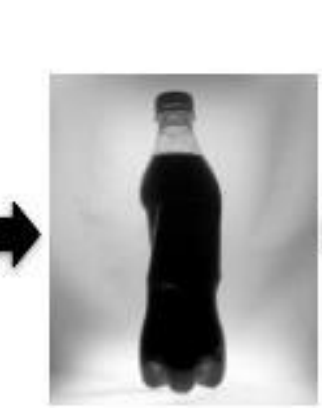

(b)

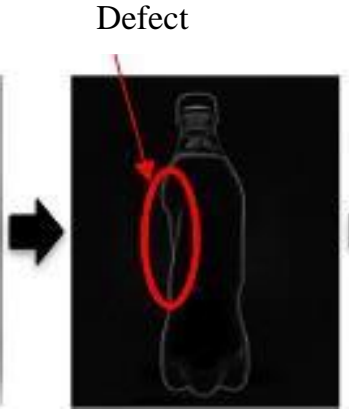

(c)

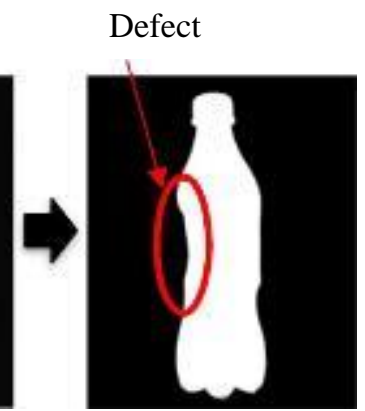

(d)

Figure 7. Shape detection process: (a) Input stage, (b) pre-processing stage, (c) LSDstage, (d) feature extraction stage

The implementation of Hough transform makes the maximum and minimum of water level is estimated using voting process. The level defect detection process is indicated in Figure 8(a) to Figure 8(d). The sample of reference image in Figure 8(a) is converted into gray scale image. Then, LSD technique is applied to segment the level of the water that is shown in Figure 8(b). Figure 8(c) illustrates the several lines created using Hough transform technique to find the maximum and minimum level value based on the white pixel of the image. The line detection image is shown in Figure $8(\mathrm{~d})$, in which the blue line presents minimum level and the red line indicates maximum level. The process is repeated for overfilled and underfilled condition as graphically shows in Figure 9(a) and Figure 9(b).

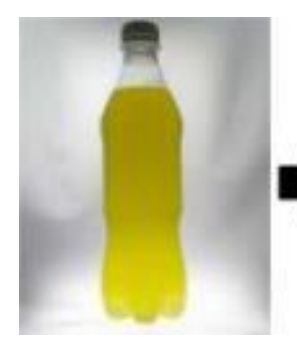

(a)

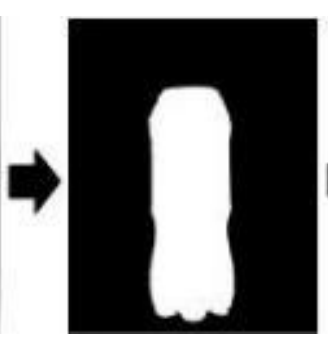

(b)

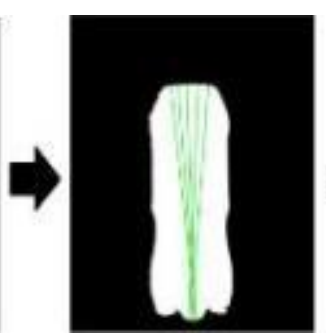

(c)

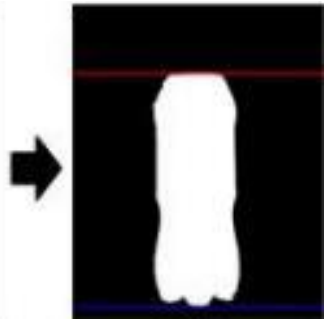

(d)

Figure 8. Good level image: (a) Sample image, (b) segmented image, (c) Hough transform image, (d) line detection image 


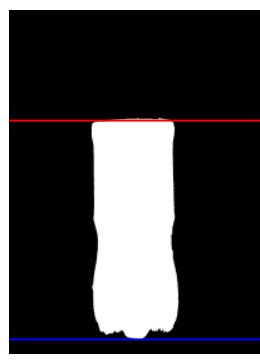

(a)

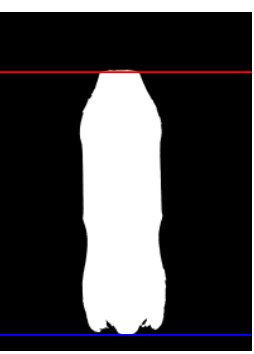

(b)

Figure 9. Level image: (a) Underfilled, (b) overfilled

In obtaining the threshold value of the water level, the distance of the water level is calculated between the maximum and minimum lines using the equation (6). Figure 10 shows three misclassified of $x$ node (red color) that plotted in scatter diagram. In defect condition, there is case where decision tree misclassified the shape of the bottle as good condition. This case will lead to inaccuracy that inappropriate for real time application. Figure 11 illustrates the scatter plot diagrams that classify the sample image based on level condition, which are good, overfilled and underfilled. Based on Figure 11, the decision tree is misclassified ofx node (blue color) as good condition. The actual class of the misclassified node is underfilled and overfilled condition.

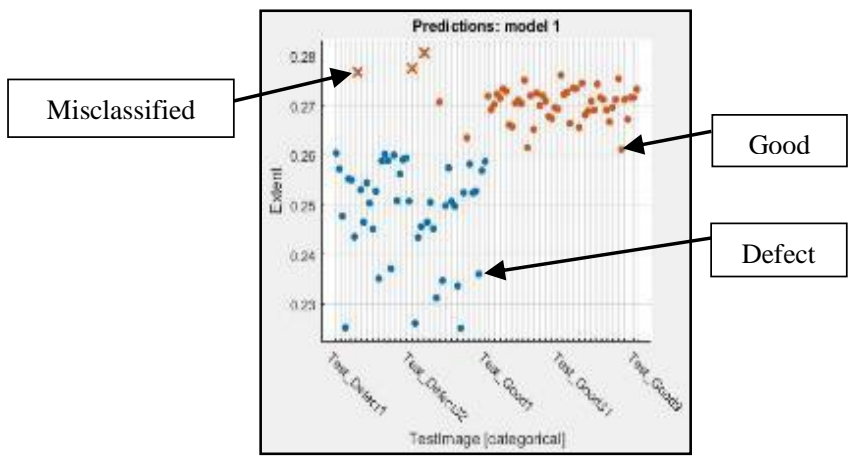

Figure 10. Scatter plot diagram of shape defect classification using LSD technique

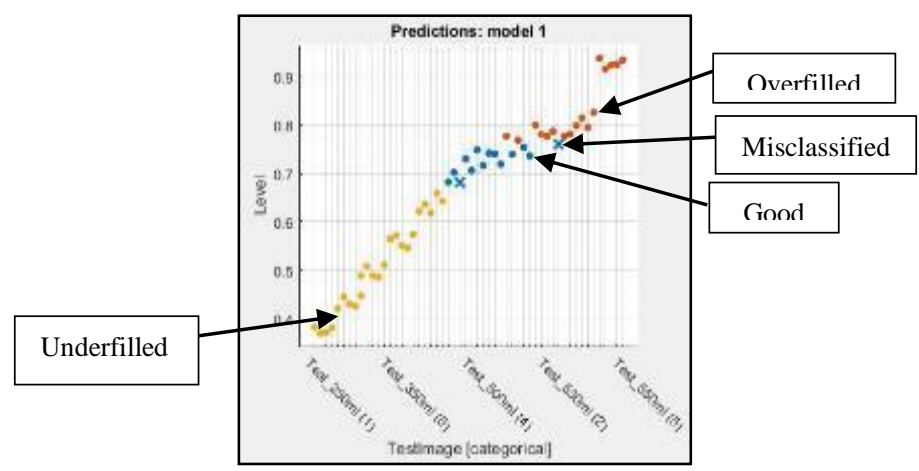

Figure 11. Scatter plot diagram of level defect classification using Hough transform

The comparison in term of accuracy for shape and level detection techniques are shown in Figure 12. From the findings, LSD technique shows the highest percentage with accuracy $97 \%$ followed by SHFCM with $91 \%$ and morphological operation with $80 \%$. In this case, the outcome using morphological operation is error prone, not to mention a large different of $17 \%$ compared to LSD. The aim for level detection is 
findingan appropriate analysis which can detect level of liquid bottle accurately.Hence, Hough transform has proven that it can perform absolutely well in detecting liquid level by achieving $93 \%$ accuracy using decision tree classifier.

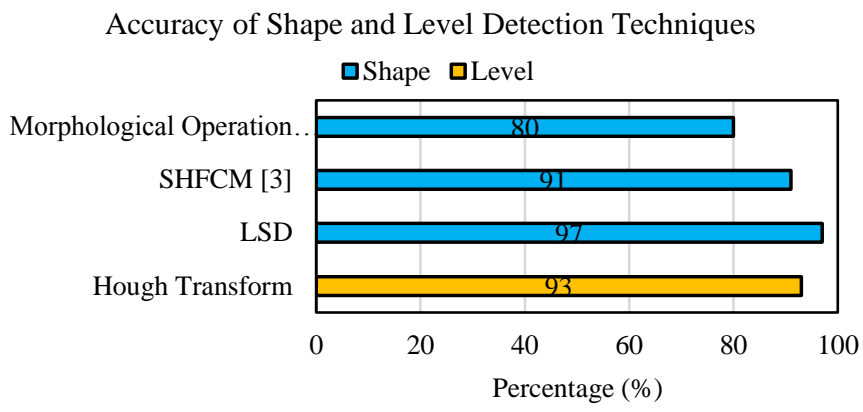

Figure 12. Performance analysis for shape and level defect detection

\section{CONCLUSION}

In this paper, the analysis of shape and level of bottle is presented using local standard deviation and Hough transform. The analysis involved pre-processing, segmentation, feature extraction and classification. The sample image is pre-processed to eliminate the noise that occurs on the image which gives complexity during segmentation process. In segmenting the image, LSD technique is applied byenhancing the contrast gain of the image from low to high. For shape features, extent parameter is calculated to determine the ratio of the bottle area. Meanwhile, Hough transform is used to extract the features of the water level such as maximum and minimum level. The decision tree classifier is applied to classify the shape and level either good or defect. The performance of the proposed techniques is verified in terms of accuracy. The experimental result shows $97 \%$ and $93 \%$ accuracy is achieved by shape and level detection. Thus, the proposed techniques demonstrate the potential to detect the shape and level for beverages product.

\section{ACKNOWLEDGEMENTS}

The authors would like to thank the Universiti Teknikal Malaysia Melaka (UTeM), UTeM Zamalah Scheme, Rehabilitation Engineering \& Assistive Technology (REAT) research group under Center of Robotics \& Industrial Automation (CeRIA), Advanced Digital Signal Processing (ADSP) Research Laboratory and Ministry of Higher Education (MOHE), Malaysia for sponsoring this work under project GLuar/STEVIA/2016/FKE-CeRIA/100009 and the use of the existing facilities to complete this project.

\section{REFERENCES}

[1] K.B. Kim, H.J. Park and D.H. Song. Vision-based Crack Identification on the Concrete Slab Surface using Fuzzy Reasoning Rules and Self-Organizing. International Journal of Electrical and Computer Engineering (IJECE), 6(4), pp.1577-1586, 2016.

[2] N.N.S.A. Rahman, Saad, N.M. Saad, A.R. Abdullah, M.R.M. Hassan, M.S.S.M. Basir and N.S.M. Noor. Automated Real-Time Vision Quality Inspection Monitoring System. Indonesian Journal of Electrical Engineering and Computer Science, 11(2), pp.775-783, 2018

[3] G. Moradi, M. Shamsi, M. H. Sedaaghi, and S. Moradi, "Apple defect detection using statistical histogram based Fuzzy C-means algorithm”, Inst. Electr. Electron. Eng., pp. 11-15, 2011.

[4] M. Park, J.S. Jin, S.L. Au, S. Luo, and Y. Cui, "Automated Defect Inspection System by Pattern Recognition", Proc. 5th Int. Conf. Image Graph. ICIG 2009, vol. 2, no. 2, pp. 768-773, 2009.

[5] M.A.M. Fuad, M.R. Ab Ghani., R. Ghazali, M.F. Sulaima, M.H. Jali, T. Sutikno, T.A. Izzuddin and Z. Jano. "A Review on Methods of Identifying and Counting Aedes Aegypti Larvae using Image Segmentation Technique". TELKOMNIKA (Telecommunication Computing Electronics and Control), 15(3), pp.1199-1206, 2017.

[6] S. Ramli, M.M. Mustafa, A. Hussain, and D.A. Wahab, "Plastic Bottle Shape Classification Using Partial Erosionbased Approach", Mod. Appl. Sci., vol. 6, no. 4, pp. 77-83, 2012.

[7] K.J. Pithadiya, C.K. Modi, and J.D. Chauhan, "Machine Vision Based Liquid Level Inspection System using ISEF Edge detection Technique", Int. Conf. Work. Emerg. Trends Technol. (ICWET), no. Icwet, pp. 601-605, 2010.

[8] L. Yazdi, A.S. Prabuwono, and E. Golkar, "Feature extraction algorithm for fill level and cap inspection in bottling machine", Proc. 2011 Int. Conf. Pattern Anal. Intell. Robot. ICPAIR 2011, vol. 1, no. June, pp. 47-52, 2011. 
[9] Y. Yang and Z. Zhou, "Research and Implementation of Image Enhancement Algorithm Based on Local Mean and Standard Deviation", IEEE Symp. Electr. Electron. Eng., pp. 375-378, 2012.

[10] O. Barinova, V. Lempitsky, and P. Kohli, "On detection of multiple object instances using Hough transform", IEEE Trans. Pattern Anal. Mach. Intell., vol. 34, no. 9, pp. 1773-1784, 2010.

[11] R. Perera and S. Premasiri, "Hardware Implementation of Essential Pre-Processing \& Morphological Operations in Image Processing”, Natl. Conf. Technol. Manag., pp. 3-7, 2017.

[12] J. Xie, Y. Zhou and L. Ding. Local standard deviation spectral clustering. In Big Data and Smart Computing (BigComp), 2018 IEEE International Conference on (pp. 242-250). IEEE. 2018, January.

[13] B.S. Prakoso, I.K. Timotius, and I. Setyawan, "Palmprint Identification for User Verification based on Line Detection and Local Standard Deviation”, 2014 1st Int. Conf. Inf. Technol. Comput. Electr. Eng. Green Technol. Its Appl. a Better Futur. ICITACEE 2014 - Proc., pp. 155-159, 2014.

[14] A. Kaur and C. Singh. Contrast enhancement for cephalometric images using wavelet-based modified adaptive histogram equalization. Applied Soft Computing, 51, pp.180-191, 2017.

[15] V.S. Tallapragada, D.M. Reddy, P.S. Kiran and D.V. Reddy. A Novel Medical Image Segmentation and Classification using Combined Feature Set and Decision Tree Classifier. International Journal of Research in Engineering and Technology, 4(9), pp.83-86, 2016.

[16] S.A. Ludwig, S. Picek and D. Jakobovic. Classification of Cancer Data: Analyzing Gene Expression Data Using a Fuzzy Decision Tree Algorithm. In Operations Research Applications in Health Care Management (pp. 327-347). Springer, Cham, 2018.

\section{BIOGRAPHIES OF AUTHORS}
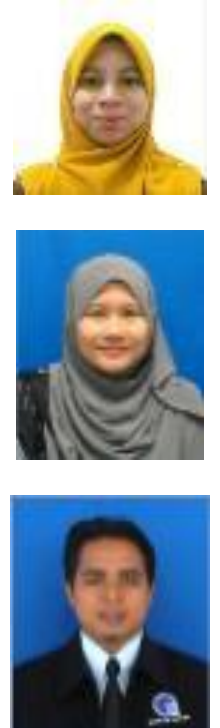

Nor Nabilah Syazana binti Abdul Rahman has received her B. Eng. from Universiti Teknikal Malaysia in 2016. She is currently pursuing her Master Eng. in Universiti Teknikal Malaysia. Her research areas are in image processing and computer vision for product quality inspection system.

Dr. Norhashimah binti Mohd Saad is currently working as a senior lecturer in Department Computer, FKEKK, UTeM. She finished her study in Bachelor of Engineering, Master of Engineering and $\mathrm{PhD}$ in Medical Image Processing from UTM, Malaysia.

Associate Prof. Dr. Abdul Rahim bin Abdullah has received his B. Eng., Master Eng., PhD Degree from Universiti Teknologi Malaysia in 2001, 2004 and 2011 in Electrical Engineering and Digital Signal Processing respectively. He is currently an Associate Professor with the Department of Electrical Engineering for Universiti Teknikal Malaysia Melaka (UTeM). 